\title{
Analytical Solution of the Perturbed Oribt-Attitude Motion of a Charged Spacecraft in the Geomagnetic Field
}

\author{
Hani M. Mohmmed*, Mostafa K. Ahmed ${ }^{\dagger}$, Ashraf Owis ${ }^{\ddagger}$ and Hany Dwidar ${ }^{\S}$ \\ * Graduate Research Assistant, Department of Solar and Space Research, NRIAG, El Marsad st. Helwan 11421, Cairo, Egypt. \\ E-mail: hani.20@yahoo.co.uk \\ $\dagger$ Cairo University, 13126 (Egypt) \\ E-mail: mkahmed.g@gmail.com \\ $\ddagger$ Cairo University, 13126 (Egypt) \\ E-mail: aowis@eun.eg \\ $\S$ Cairo University, 13126 (Egypt) \\ E-mail: hrydwidar@gmail.com
}

\begin{abstract}
In this work we investigate the orbit-attitude perturbations of a rigid spacecraft due to the effects of several forces and torques. The spacecraft is assumed to be of a cylindrical shape and equipped with a charged screen with charge density $\sigma$. Clearly the main force affecting the motion of the spacecraft is the gravitational force of the Earth with uniform spherical mass. The effect of oblate Earth up to $J_{2}$ is considered as perturbation on both the orbit and attitude of the spacecraft, where the attitude of the spacecraft is acted upon by what is called gravity gradient torque. Another source of perturbation on the attitude of the spacecraft comes from the motion of the charged spacecraft in the geomagnetic field. This motion generates a force known as the Lorentz force which is the source of the Lorentz force torque influencing the rotational motion of the spacecraft. In this work we give an analytical treatment of the orbital-rotational dynamics of the spacecraft. We first use the definitions of Delaunay and Andoyer variables in order to formulate the Hamiltonian of the orbit-attitude motion under the effects of forces and torques of interest. Since the Lorentz force is a non-conservative force, a potential like function is introduced and added to the Hamiltonian. We solve the canonical equations of the Hamiltonian system by successive transformations using a technique proposed by Lie and modified by Deprit and Kamel to solve the problem. In this technique we make two successive transformations to eliminate the short and long periodic terms from the Hamiltonian.
\end{abstract}

\section{INTRODUCTION}

The motion of a rigid spacecraft is specified by its position, velocity, attitude, and attitude motion. The first two describe the translational motion of the center of mass of the spacecraft, while the latter two describe the rotational motion of the body about the center of mass, In general the translational and attitude motions are independent as long as no resonance conditions are assumed between the orbital and rotational motions, where in this case attitude-orbit coupling results. A spacecraft is in general under the perturbation effect of gravitaional potential of the Earth, which includes both perturbations on the orbital and the attitude motion of the spacecraft. Other forces maybe added to the gravitational force, such as solar radiation pressure, which is investigated analytically in [11] and [2] . In this work we invesitgate analytically the perturbation effects of both the gravitational force up to $J_{2}$ and Lorentz force on both the orbital and attitude motion. Following the work of [2], this paper is organized as follows: we formulate the Hamiltonian of the motion of the spacecraft under the perturbing forces and torques, the problem is then tackled using the straight forward Lie technique. This technique was proposed by Lie and developed by Deprit and Kamel (see [1] and [5]). In this technique we perform two successive transformations to eliminate the short and long periodic terms from the Hamiltonian, and hence the new canonical equations are solved easily. The novelty of this work is that we obtain an analytical solution of the problem. Despite the numerical solution is accurate and can be applied in practise, analytical treatments can lead to closed form solutions, and enables us to analyze the problem.

\section{COORDINATE SYSTEMS AND SYSTEMS OF CANONICAL VARIABLES}

Before starting to formulate the problem we first define the coordinate systems and the canonical variables used to describe the motion. Let the inertial coordiante system $O X Y Z$ with origin at the Earth's center and defined such that the $X$-axis is toward the Vernal Equinox, the $Y$-axis is normal to the $X$-axis and located in the equatorial plane and the $Z$-axis coincides with the rotational axis of the Earth. Three unit vectors $(\hat{i}, \hat{j}, \hat{k})$ are taken in the $X, Y, Z$ directions respectively. The body coordinate System $O^{\prime} X^{\prime} Y^{\prime} Z^{\prime}$ is located at the center of mass of the spacecraft with $X^{\prime}$-axis, $Y^{\prime}$-axis and $Z^{\prime}$-axis choosen along the principal axes of the spacecraft.The three unit vectors $\left(\hat{e_{1}}, \hat{e_{2}}, \hat{e_{3}}\right)$ are taken in the $X^{\prime}, Y^{\prime}, Z^{\prime}$ directions respectively. The orbital coordinate system $O^{\prime} \xi \eta \zeta$ is located at the center of mass of the spacecraft and with the $\xi$-axis 
along the radius vector of the spacecraft from the geocenter, the $\eta$-axis is normal to the radius vector and in located in the orbital plane and $\zeta$-axis is normal to the orbital plane.The three unit vectors $(\hat{R}, \hat{S}, \hat{T})$ are taken in the $\xi, \eta, \zeta$ directions respectively.

\section{A. Sets of Canonical variables}

There are two types of sets of canonical variables used, one for the description of the orbital motion, and the other describes the attitude motion. There are many sets of variables for both types. Two of the most famous sets are Delaunay and Andoyer sets of variables used to describe both orbital and attitude motion respectively.

\section{B. Delaunay variables}

Delaunay variables are usually used to describe the orbital motions of the Earth and of the Moon and Sun, assumed to produce measurable tidal effects. They are usually defined as

$l$ : Mean anomaly, $g$ : Argument of perigee, $h$ : Longitude of the node, $L=\sqrt{\mu a}, G=\sqrt{\mu a\left(1-e^{2}\right)}$ and $H=$ $\sqrt{\mu a\left(1-e^{2}\right)} \cos I$. Where $\mu$ is the Earth's mass, $a$ is the semi-major axis of the spacecraft, $e$ is the eccenricity, and $I$ is the inclination.

\section{Andoyer variables}

Andoyer variables are the most commonly used for the description of attitude motion. [3] performed transformation from the inertial frame to the body frame through the intermediate invariable plane using $3-1-3-1-3$ successive rotations.

The transformation matrix of this transformation is given in terms of Andoyer variables as:

$$
R\left(h_{a}, I_{a}, g_{a}, J_{a}, l_{a}\right)=\left[\begin{array}{lll}
\boldsymbol{V}_{1} & \boldsymbol{V}_{2} & \boldsymbol{V}_{3}
\end{array}\right]
$$

where $I_{a}$ is the inclination of the invariable plane on the reference plane, $J_{a}$ is the angle between the invariable plane and the body plane. The column vectors $\boldsymbol{V}_{1}, \boldsymbol{V}_{2}$ and $\boldsymbol{V}_{3}$ are given as:

$$
\begin{gathered}
\boldsymbol{V}_{1}=\left[\begin{array}{c}
\left(c_{h_{a}}\left(c_{g_{a}} c_{l_{a}}-c_{J_{a}} s_{g_{a}} s_{l_{a}}\right)-s_{h_{a}}\left(-s_{I_{a}} s_{J_{a}} s_{l_{a}}\right.\right. \\
\left.+c_{I_{a}}\left(c_{l_{a}} s_{g_{a}}+c_{g_{a}} c_{J_{a}} s_{l_{a}}\right)\right) \\
c_{h_{a}}\left(-c_{J_{a}} c_{l_{a}} s_{g_{a}}-c_{g_{a}} s_{l_{a}}\right)-s_{h_{a}}\left(-c_{l_{a}} s_{I_{a}} s_{J_{a}}\right. \\
\left.+c_{I_{a}}\left(c_{g_{a}} c_{J_{a}} c_{l_{a}}-s_{g_{a}} s_{l_{a}}\right)\right) \\
c_{h_{a}} s_{g_{a}} s_{J_{a}}-s_{h_{a}}\left(-c_{J_{a}} s_{I_{a}}-c_{I_{a}} c_{g_{a}} s_{J_{a}}\right)
\end{array}\right] \\
\boldsymbol{V}_{2}=\left[\begin{array}{c}
s_{h_{a}}\left(c_{g_{a}} c_{l_{a}}-c_{J_{a}} s_{g_{a}} s_{l_{a}}\right) \\
+c_{h_{a}}\left(-s_{I_{a}} s_{J_{a}} s_{L_{a}}+c_{I_{a}}\left(c_{l_{a}} s_{g_{a}}+c_{g_{a}} c_{J_{a}} s_{l_{a}}\right)\right) \\
s_{h_{a}}\left(-c_{J_{a}} c_{l_{a}} s_{g_{a}}-c_{g_{a}} s_{l_{a}}\right) \\
+c_{h_{a}}\left(-c_{l_{a}} s_{I_{a}} s_{J_{a}}+c_{I_{a}}\left(c_{g_{a}} c_{J_{a}} c_{L_{a}}-s_{g_{a}} s_{l_{a}}\right)\right) \\
s_{g_{a}} s_{h_{a}} s_{J_{a}}+c_{h_{a}}\left(-c_{J_{a}} s_{I_{a}}-c_{I_{a}} c_{g_{a}} s_{J_{a}}\right)
\end{array}\right] \\
\boldsymbol{V}_{3}=\left[\begin{array}{c}
c_{I_{a}} s_{J_{a}} s_{l_{a}}+s_{I_{a}}\left(c_{l_{a}} s_{g_{a}}+c_{g_{a}} c_{J_{a}} s_{l_{a}}\right) \\
c_{I_{a}} c_{l_{a}} s_{J_{a}}+s_{I_{a}}\left(c_{g_{a}} c_{J_{a}} c_{l_{a}}-s_{g_{a}} s_{l_{a}}\right) \\
c_{I_{a}} c_{J_{a}}-s_{I_{a}} s_{J_{a}} c_{g_{a}}
\end{array}\right]
\end{gathered}
$$

Andoyer variables used in the previous transformation are defined as: $L_{a} \equiv Z^{\prime}$ component of the angular momentum vector (i.e. that normal to the body plane), $G_{a} \equiv$ Total angular momentum vector, $H_{a} \equiv Z$ component of the angular momentum vector (i.e. that normal to the reference plane). The conjugate angle variables are :

$l_{a} \equiv$ the longitude of the $X^{\prime}$ axis (of the body frame) with respect to the node of the body plane on the invariable plane. $g_{a} \equiv$ the longitude of the node $N_{3}$ with respect to $N_{2}$ (measured in the invariable plane).

$h_{a} \equiv$ longitude of the node $N_{2}$ with respect to the $X$ axis (of the inertial frame).

\section{FORMULATION OF THE HAMILTONIAN OF THE ORBIT-ATTITUDE MOTION}

The Hamiltonian of the orbit-attitude motion of the spacecraft is formulated using the prescribed Delaunay and Andoyer variables. The total Hamiltonian consists of the Hamiltonian of the gravitational potential, the Hamiltonian of the torque free motion, and the Hamiltonian of the Lorentz torques.

\section{A. Hamiltonian of the gravitational potential}

As mentioned before the gravitational potential of oblate Earth will be considered affecting on a spacecraft of a cylindrical shape and consisting of only one single body. The gravitational potential of the Earth is given by [4] as

$$
\begin{aligned}
V_{g}= & \left.-\frac{\mu m}{R_{c}}\left\{1+\frac{1}{2} J_{2}\left(\frac{R_{e}}{R_{c}}\right)^{2}\left(1-3 \sin ^{2} \phi_{c}\right)\right\} \Sigma_{2}\right) \\
& -\frac{\mu}{2 R_{c}^{3}}\left\{\left(\Sigma_{1}-3+\frac{3}{2} J_{2}\left(\frac{R_{e}}{R_{c}}\right)^{2} \times\left[\left(1-5 \sin ^{2} \phi_{c}\right) \Sigma_{1}\right.\right.\right. \\
& \left.\left.-5\left(1-7 \sin ^{2} \phi_{c}\right) \Sigma_{2}+2 \Sigma_{3}+20 \sin \phi_{c} \Sigma_{4}\right]\right\}
\end{aligned}
$$

where $\sin \phi_{c}=\sin I \sin (f+g), J_{2}=1.083 \times 10^{-3}$ is the coefficient of the second harmonic of Earth's gravitational potential and

$\Sigma_{1}=\sum_{i=1}^{3} I_{i}, \Sigma_{2}=\sum_{i=1}^{3} I_{i} c_{i 3}^{2}, \Sigma_{3}=\sum_{i=1}^{3} I_{i} c_{e i 3}^{2}, \Sigma_{4}=\sum_{i=1}^{3} I_{i} c_{i 3} c_{e i 3}$

and $I_{i}$ are the principle moments of inertia of the satellite, $c_{i 3}$ are the direction cosines between the principle axes and the unit vector $\hat{S}$ along the radius vector $\mathbf{R}_{\mathbf{c}}$, and $c_{e i 3}$ are the direction cosines between the principal axes and the Earth's rotational axis.

The direction cosines $c_{i 3}$ and $c_{e i 3}$ need to be evaluated in terms of Delaunay and Andoyer variables. They were evaluated in [2] and [6] as: 


$$
\begin{aligned}
& c_{13}= \sum_{\substack{i, j, k=-1 \\
k \neq 0}}^{1} A_{i j k} \cos \left((f+g)+i\left(h_{a}-h\right)+j g_{a}+k l_{a}\right) \\
& c_{23}=\sum_{\substack{i, j, k=-1 \\
k \neq 0}}^{1} B_{i j k} \sin \left((f+g)+i\left(h_{a}-h\right)+j g_{a}+k l_{a}\right) \\
&
\end{aligned}
$$

and

$$
c_{33}=\sum_{i, j=-1}^{1} C_{i j} \sin \left((f+g)+i\left(h_{a}-h\right)+j g_{a}\right)
$$

where the coefficients $A_{i j k}, B_{i j k}$ and $C_{i j}$ are known functions of $I, I_{a}$ and $J_{a}$ arising from the transformations between frames. Now after evaluating the direction $\operatorname{cosines} c_{i 3}$ we proceed in evaluating their squares $c_{i 3}^{2}$. We have

$$
c_{13}^{2}=\sum_{i=0,2} \sum_{j, k, m=-2}^{2} P_{i j k m} \cos \left(i(f+g)+j\left(h_{a}-h\right)+k g_{a}+m l_{a}\right)
$$

where

$$
\begin{gathered}
P_{0 j k m}=P_{2 j k m}=\sum_{\substack{\nu_{1}, \mu_{1}=-1 \\
\nu_{1} \pm \mu_{1}=j}}^{1} \sum_{\substack{\nu_{2}, \mu_{2}=-1 \\
\nu_{2} \pm \mu_{2}=k}}^{1} \sum_{\substack{\nu_{3}, \mu_{3}=-1,1 \\
\nu_{3} \pm \mu_{3}=m}} \frac{A_{\nu_{1} \nu_{2} \nu_{3}} A_{\mu_{1} \mu_{2} \mu_{3}}}{2} \\
c_{23}^{2}=\sum_{i=0,2}^{2} \sum_{j, k, m=-2} Q_{i j k m} \cos \left(i(f+g)+j\left(h_{a}-h\right)+k g_{a}+m l_{a}\right)
\end{gathered}
$$

where

$$
\begin{aligned}
& Q_{0 j k m}=Q_{2 j k m}=\sum_{\substack{\nu_{1}, \mu_{1}=-1 \\
\nu_{1}-\mu_{1}=j}}^{1} \sum_{\substack{\nu_{2}, \mu_{2}=-1 \\
\nu_{2}-\mu_{2}=k}}^{1} \underset{\substack{\nu_{3}, \mu_{3}=-1,1 \\
\nu_{3}-\mu_{3}=m}}{\frac{B_{\nu_{1} \nu_{2} \nu_{3}} B_{\mu_{1} \mu_{2} \mu_{3}}}{2}} \\
& \begin{array}{ccc}
-\sum_{\substack{1 \\
\nu_{1}, \mu_{1}=-1 \\
\nu_{1}+\mu_{1}=j}} \sum_{\substack{\nu_{2}, \mu_{2}=-1 \\
\nu_{2}+\mu_{2}=k}} \sum_{\nu_{3}, \mu_{3}=-1,1} \frac{B_{\nu_{1} \nu_{2} \nu_{3}} B_{\mu_{1} \mu_{2} \mu_{3}}}{2} \\
\nu_{3}+\mu_{3}=m
\end{array}
\end{aligned}
$$

and

$$
c_{33}^{2}=\sum_{i=0,2} \sum_{j, k=-2}^{2} R_{i j k} \cos \left(i(f+g)+j\left(h_{a}-h\right)+k g_{a}\right)
$$

where

$$
\begin{gathered}
R_{0 j k}=R_{2 j k}=\sum_{\substack{\nu_{1}, \mu_{1}=-1 \\
\nu_{1}-\mu_{1}=j}}^{1} \sum_{\substack{\nu_{2}, \mu_{2}=-1 \\
\nu_{2}-\mu_{2}=k}}^{1} \frac{C_{\nu_{1} \nu_{2}} C_{\mu_{1} \mu_{2}}}{2} \\
-\sum_{\sum_{1}, \mu_{1}=-1}^{1} \sum_{\nu_{2}, \mu_{2}=-1} \frac{C_{\nu_{1} \nu_{2}} C_{\mu_{1} \mu_{2}}}{2} \\
\nu_{1}+\mu_{1}=j \\
\nu_{2}+\mu_{2}=k
\end{gathered}
$$


The direction cosines $c_{e i 3}$ are evaluated as:

$$
\begin{gathered}
c_{e 13}=\sum_{\substack{i=0, j=-1}}^{1} A_{e i j} \sin \left(i g_{a}+j l_{a}\right) \\
c_{e 23}=\sum_{\substack{i=0, j=-1}}^{1} B_{e i j} \cos \left(i g_{a}+j l_{a}\right) \\
c_{e 33}=\sum_{i=0}^{1} C_{e i} \cos \left(i g_{a}\right)
\end{gathered}
$$

Here $A_{e i j}, B_{e i j}$ and $C_{e i}$ are functions of $I, I_{a}$, and $J_{a}$. Their squares are also evaluated as:

$$
c_{e 13}^{2}=\sum_{j=-1, k=-2}^{2} E_{j k} \cos \left(j g_{a}+k l_{a}\right)
$$

where

$$
\begin{aligned}
& E_{j k}=\sum_{\substack{\nu_{1}, \mu_{1}=0 \\
\nu_{1}-\mu_{1}=j}}^{1} \sum_{\substack{\nu_{2}, \mu_{2}=-1 \\
\nu_{2}-\mu_{2}=k}}^{1} \frac{A_{e \nu_{1} \nu_{2}} A_{e \mu_{1} \mu_{2}}}{2} \\
& \begin{array}{ccc}
-\sum_{\substack{\nu_{1}, \mu_{1}=0 \\
\nu_{1}+\mu_{1}=j}}^{1} & \sum_{\substack{\nu_{2}, \mu_{2}=-1 \\
\nu_{2}+\mu_{2}=k}}^{1} \frac{A_{e \nu_{1} \nu_{2}} A_{e \mu_{1} \mu_{2}}}{2}
\end{array} \\
& c_{e 23}^{2}=\sum_{j=-1, k=-2}^{2} F_{j k} \cos \left(j g_{a}+k l_{a}\right)
\end{aligned}
$$

where

$$
F_{j k}=\sum_{\substack{\nu_{1}, \mu_{1}=0 \\ \nu_{1} \pm \mu_{1}=j}}^{1} \sum_{\substack{\nu_{2}, \mu_{2}=-1 \\ \nu_{2} \pm \mu_{2}=k}}^{1} \frac{B_{e \nu_{1} \nu_{2}} B_{e \mu_{1} \mu_{2}}}{2}
$$

and

$$
c_{e 33}^{2}=\sum_{j=-1}^{2} G_{j} \cos \left(j g_{a}\right)
$$

where

$$
G_{j}=\sum_{\substack{\nu_{1}, \mu_{1}=0 \\ \nu_{1} \pm \mu_{1}=j}}^{1} \frac{C_{e \nu_{1}} C_{e \mu_{1}}}{2}
$$

The products $c_{i 3} c_{e i 3}$ are also evaluated as:

$$
c_{13} c_{e 13}=\sum_{j=-1}^{1} \sum_{k, m=-2}^{2} U_{j k m} \sin \left((f+g)+j\left(h_{a}-h\right)+k g_{a}+m l_{a}\right)
$$


where

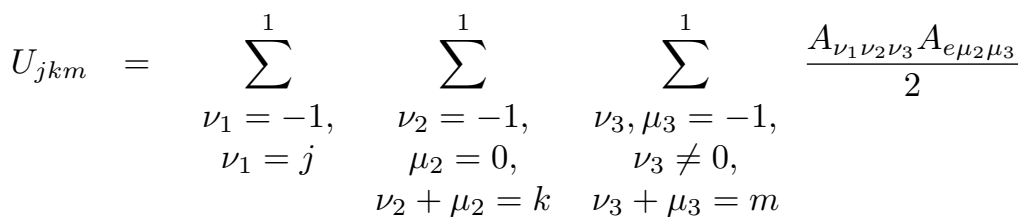

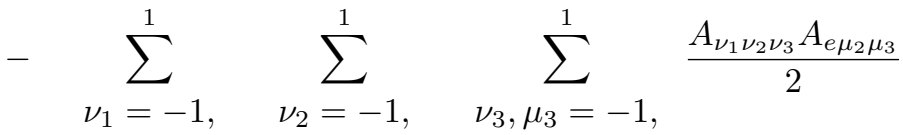

$$
\begin{aligned}
& \nu_{1}=j \quad \mu_{2}=0, \quad \nu_{3} \neq 0, \\
& \nu_{2}-\mu_{2}=k \quad \nu_{3}-\mu_{3}=m
\end{aligned}
$$

In the same way we get :

$$
c_{23} c_{e 23}=\sum_{j=-1}^{1} \sum_{k, m=-2}^{2} V_{j k m} \sin \left((f+g)+j\left(h_{a}-h\right)+k g_{a}+m l_{a}\right)
$$

where

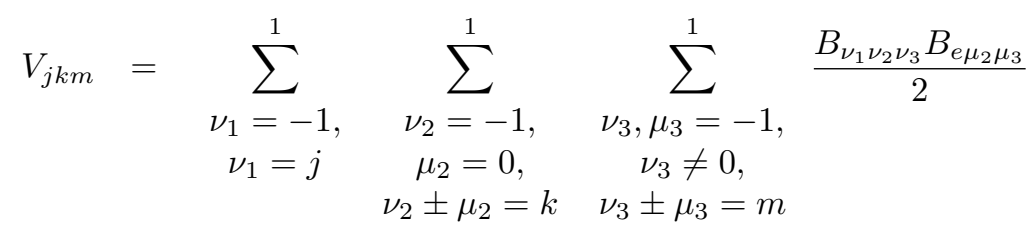

and

$$
c_{33} c_{e 33}=\sum_{j=-1}^{1} \sum_{k=-2}^{2} W_{j k} \sin \left((f+g)+j\left(h_{a}-h\right)+k g_{a}\right)
$$

where

$$
W_{j k}=\sum_{\substack{\nu_{1}=-1, \nu_{1}=j}}^{1} \sum_{\substack{\nu_{2}=-1, \mu_{2}=0, \nu_{2} \pm \mu_{2}=k}}^{1} \frac{C_{\nu_{1} \nu_{2}} C_{e \mu_{2}}}{2}
$$

\section{B. Contribution of the gravitational potential to the total Hamiltonian}

Considering the spacecraft is of a cylindrical shape and considering the dimensions of the cylinder to be of length $\approx 100 \mathrm{~m}$ then $\frac{\rho}{R_{E}}$ is of order $J_{2}$, where $\rho$ is the radius vector of any point on the spacecraft relative to its center of mass, and $R_{E}$ is the Earth's radius. Now note that the principal moments of inertia $I_{i}$ are directly proportional to the square of $\rho$, $\sin ^{2}\left(\phi_{c}\right)=\sin ^{2}(I)\left(\frac{1}{2}-\frac{1}{2} \cos (2 g+2 f)\right)$ and $a=\frac{L^{2}}{\mu}$. We then have a new form of the gravitational potential written in terms of Delaunay and Andoyer variables as:

$$
\begin{aligned}
V_{g} & =-\frac{\mu^{2}}{2 L^{2}}+\frac{1}{4} \frac{A_{2}}{L^{6}}\left(\frac{a}{R_{c}}\right)^{3}\left[\left(3 s^{2}-2\right)-3 s^{2} \cos (2 f+2 g)\right] \\
- & \frac{J_{2}^{2}}{2 !} \frac{\mu^{4} R_{e}^{2}}{2 L^{6}}\left(\frac{a}{R_{c}}\right)^{3}\left[\sum_{\substack{j, k, m=-2 \\
i=0.2}}^{j} \widetilde{B}_{i j k m}^{11} \cos \left(i(f+g)+j\left(h_{a}-h\right)+k g_{a}+m l_{a}\right)\right] \\
- & \frac{J_{2}^{3}}{3 !} \frac{3 \mu^{6} R_{e}^{4}}{2 L^{10}}\left(\frac{a}{R_{c}}\right)^{5}\left[\sum_{\substack{j, k, m=2 \\
j, k=-2}}^{\substack{j, k, m=2,0,2 \\
i=-2,0}} \widetilde{C}_{i j k m}^{11} \cos \left(i(f+g)+j\left(h_{a}-h\right)+k g_{a}+m l_{a}\right)\right]
\end{aligned}
$$

where $R_{c}$ is the distance from the center of Earth to the center of mass of the spacecraft, $s=\sin I, A_{2}=\mu^{4} R_{e}^{2}$ and the coefficients $\widetilde{B}_{i j k m}^{11}$ and $\widetilde{C}_{i j k m}^{11}$ are functions of $I_{a}, J_{a}, I, I_{i}, R_{e}$ and $J_{2}$ 


\section{Contribution of Lorentz force to the Hamiltonian}

If we consider the spacecraft is equipped with a charged cylindrical sheet with surface charge density $\sigma$, the total charge of the spacecraft is $Q=\int_{S} \sigma d S$, where $S$ is the surface area of the screen. It was shown by [?] that when the center of charge is off-center, the expression for Lorentz torque can be approximated by :

$$
\mathbf{N}_{l}=Q \rho_{\circ} \times(\mathbf{v} \times \mathbf{B})_{b}
$$

where $\rho_{\circ}$ is the radius vector of the center of charge and is given by in the body coordinate system

$$
\rho_{\circ}=x_{\circ} \hat{e_{1}}+y_{\circ} \hat{e_{2}}+z_{\circ} \hat{e_{3}}=\frac{1}{Q} \int_{S} \sigma \rho d S
$$

$\mathbf{v}$ is the velocity of the center of mass of the spacecraft relative to the ECEF (Earth Centered Earth Fixed) frame and $\mathbf{B}$ is the geomagnetic field intensity.

$$
\mathbf{B}=-\operatorname{grad} U_{m}
$$

where $U_{m}$ is the geomagnetic potential. We use here the inclined dipole model for the geomagnetic potential described in the Cartesian coordinates. [?]

$$
U_{m}=\frac{R_{e}^{3}}{R_{c}^{2}}\left(g_{0} \frac{z}{R_{c}}+g_{1} \frac{x}{R_{c}}+h_{1} \frac{y}{R_{c}}\right)
$$

where

$$
g_{0}=-29615, g_{1}=-1728, h_{1}=5186
$$

Evaluating equation (18) in terms of Delaunay and Andoyer variables takes long calculations. The final form of the Lorentz torque is then

$$
\begin{aligned}
& \mathbf{N}_{l}=Q\left(\frac{R_{E}}{R_{c}}\right)^{3}\left[\left(\sum _ { \substack { i , j = 0 \\
k = - 2 } } ^ { i , j = 3 , k = 2 } \sum _ { \substack { m , n = - 1 \\
r , s = - 1 } } ^ { 1 } \left[X_{i j k m n r s}^{0}\right.\right.\right. \\
& \times \cos \left(i f+j g+k h+m g_{a}+n h_{a}+r l_{a}+s E\right) \\
& \left.\left.+\widetilde{X}_{i j k m n r s}^{0} \sin \left(i f+j g+k h+m g_{a}+n h_{a}+r l_{a}+s E\right)\right]\right) \hat{e_{1}} \\
& +\left(\sum _ { \substack { i , j = 0 \\
k = - 2 } } ^ { i , j = 3 , k = 2 } \sum _ { \substack { m , n = - 1 \\
r , s = - 1 } } ^ { 1 } \left[Y_{i j k m n r s}^{0} \cos \left(i f+j g+k h+m g_{a}+n h_{a}+r l_{a}+s E\right)\right.\right. \\
& \left.\left.+\widetilde{Y}_{i j k m n r s}^{0} \sin \left(i f+j g+k h+m g_{a}+n h_{a}+r l_{a}+s E\right)\right]\right) \hat{e_{2}} \\
& +\left(\sum _ { \substack { i , j = 0 \\
k = - 2 } } ^ { i , j = 3 , k = 2 } \sum _ { \substack { m , n = - 1 \\
r , s = - 1 } } ^ { 1 } \left[Z_{i j k m n r s}^{0} \cos \left(i f+j g+k h+m g_{a}+n h_{a}+r l_{a}+s E\right)\right.\right. \\
& \left.\left.+\widetilde{Z}_{i j k m n r s}^{0} \sin \left(i f+j g+k h+m g_{a}+n h_{a}+r l_{a}+s E\right)\right]\right) \hat{e_{3}}
\end{aligned}
$$

where $E$ is the eccentric anomaly, and the coefficients $X_{i j k m n r s}^{0}, \widetilde{X}_{i j k m n r s}^{0}, Y_{i j k m n r s}^{0}, \widetilde{Y}_{i j k m n r s}^{0} Z_{i j k m n r s}^{0}$ and $\widetilde{Z}_{i j k m n r s}^{0}$ are known functions of $I, I_{a}$ and $J_{a}$ and arise from the transformations between coordinate systems.

\section{Potential like function}

Since Lorentz force is not conservative, i.e not derived from a potential function, we introduce the potential like function to contribute to the Hamiltonian. The potential like function $\vartheta_{l}$ has the form

$$
\vartheta_{l}=-\mathbf{N}_{\mathbf{l}} \cdot \mathbf{R}_{\mathbf{c}}=-\mathbf{N}_{\mathbf{l}} \cdot R_{c}\left(c_{13} \hat{e_{1}}+c_{23} \hat{e_{2}}+c_{33} \hat{e_{3}}\right)
$$


The potential like function is then evaluated in the form :

$$
\begin{aligned}
& \vartheta_{l}=-Q \frac{R_{E}^{3}}{R_{c}^{2}}\left(\sum _ { \substack { i , j = 4 , k = 3 \\
i , j = - 2 } } ^ { \substack { s = 1 \\
k = - 3 , r = 2 \\
k = - n = - 2 } } \left[A_{i j k m n r s}^{11}\right.\right. \\
& \times \sin \left(i f+j g+k h+m g_{a}+n h_{a}+r l_{a}+s E\right) \\
& \left.\left.+\widetilde{A}_{i j k m n r s}^{11} \cos \left(i f+j g+k h+m g_{a}+n h_{a}+r l_{a}+s E\right)\right]\right)
\end{aligned}
$$

According to [?], the magnitude of the Lorentz torque acting on a spacecraft is

$$
\left|\mathbf{N}_{l}\right|=Q\left|\rho_{\circ}\right| R_{c}\left(\omega_{\circ}-\omega_{E}\right)|\mathbf{B}|
$$

where $\omega_{\circ}$ is the angular velocity of the rotation of the orbital coordinate system relative to the inertial system and $\omega_{E}$ is the angular velocity of diurnal rotation of the Earth. Thus if we take a practical example in which $R_{c}=7 \times 10^{6} \mathrm{~m}, S=100 \mathrm{~m}^{2}$, the electric potential $U=3 \times 10^{5} \mathrm{~V}$ and electric capacity $c_{s}=10^{-11}$, so that the charge has a magnitude of $3 \times 10^{-3}$. $\omega_{\circ}=1.1 \times 10^{-3}$ and if $\left|\rho_{\circ}\right|=0.5 \mathrm{~m}$ then the Lorentz torque is of order $\sim 10^{-5}$, i.e. the order of Lorentz torque is close to $J_{2}^{2}$. In terms of the small parameter $J_{2}$ the potential like function can be written as:

$$
\begin{aligned}
& \vartheta_{l}=-\frac{J_{2}^{2}}{2 !}\left(\sum _ { \substack { s = 1 \\
i , j = - 2 \\
k = - 3 } } ^ { \substack { i , j = 4 , k = 3 \\
m , n = 2 \\
s = - 1 } } \left[A_{i j k m n r s}^{11} \sin \left(i f+j g+k h+m g_{a}+n h_{a}+r l_{a}+s E\right)\right.\right. \\
& \left.\left.+\widetilde{A}_{i j k m n r s}^{11} \cos \left(i f+j g+k h+m g_{a}+n h_{a}+r l_{a}+s E\right)\right]\right)\left(\frac{a}{R_{c}}\right)^{2} \frac{\widetilde{Q} \mu R_{E}^{3}}{L^{2}}
\end{aligned}
$$

where $\widetilde{Q}=\frac{2 ! Q}{J_{2}^{2}}$.

\section{E. Total Hamiltonian}

Using the expressions of the gravitational potential and the potential like function, the total Hamiltonian is then written as:

$$
\mathcal{H}=\sum_{n=0}^{3} \frac{J_{2}^{n}}{n !} \mathcal{H}_{n}
$$

where

$$
\mathcal{H}_{0}=-\frac{\mu^{2}}{2 L^{2}}+\frac{1}{2}\left(\frac{1}{I_{3}}-\frac{1}{I_{1}}\right) L_{a}^{2}+\frac{1}{2 I_{1}} G_{a}^{2}
$$

where the second and third terms in $\mathcal{H}_{0}$ belong to the Hamiltonian of the torque free motion.

$$
\mathcal{H}_{1}=\frac{1}{4} \frac{A_{2}}{L^{6}}\left(\frac{a}{R_{c}}\right)^{3}\left[\left(3 s^{2}-2\right)-3 s^{2} \cos (2 f+2 g)\right]
$$




$$
\begin{aligned}
& \mathcal{H}_{2}=-\frac{\mu^{4} R_{e}^{2}}{2 L^{6}}\left(\frac{a}{R_{c}}\right)^{3} \sum_{\substack{j, k, m=-2 \\
i=0.2}}^{j, k, m=2} \widetilde{B}_{i j k m}^{11} \cos \left(i(f+g)+j\left(h_{a}-h\right)+k g_{a}+m l_{a}\right) \\
& -\frac{\widetilde{Q} \mu R_{E}^{3}}{L^{2}}\left(\frac{a}{R_{c}}\right)^{2}\left(\sum _ { \substack { i , j = - 2 \\
k = - 3 } } ^ { \substack { s = 1 \\
\begin{subarray} { c } { i , j = 4 , k = 3 \\
s = - n = - 2 \\
s = - 1 } } \end{subarray} } \left[A_{i j k m n r s}^{11} \times\right.\right. \\
& \sin \left(i f+j g+k h+m g_{a}+n h_{a}+r l_{a}+s E\right) \\
& \left.\left.+\widetilde{A}_{i j k m n r s}^{11} \cos \left(i f+j g+k h+m g_{a}+n h_{a}+r l_{a}+s E\right)\right]\right) \\
& \mathcal{H}_{3}=-\frac{3 \mu^{6} R_{e}^{4}}{2 L^{10}}\left(\frac{a}{R_{c}}\right)^{5}\left[\sum_{\substack{j, k, m=-2 \\
i=-2,0,2,4}}^{j, k, m=2} \widetilde{C}_{i j k m}^{11} \cos \left(i(f+g)+j\left(h_{a}-h\right)+k g_{a}+m l_{a}\right)\right]
\end{aligned}
$$

From the above equations we note that $\mathcal{H}_{0}$ is a function of $L, L_{a}$ and $G_{a}$, hence $l, l_{a}$ and $g_{a}$ are fast variables while the other variables are slow ones.

\section{PERTURBATION APPROACH}

Let $\epsilon$ be the small parameter of the problem and let the canonical system of differential equations be written as :

$$
\dot{u}=H_{U}^{T} \quad, \quad \dot{U}=-H_{u}^{T}
$$

What is required is to construct two (or more) transformations $(u, U ; \epsilon) \rightarrow(\grave{u}, \grave{U})$ and $(\grave{u}, \grave{U} ; \epsilon) \rightarrow(\grave{\grave{u}}, \grave{U})$ analytic in $\epsilon$ at $\epsilon=0$ to eliminate in succession the short and long period terms from the Hamiltonian such that $\dot{U}$ reduce to constants and $\grave{\grave{u}}$ become linear functions of time.

The old and new Hamiltonians and the Generators are assumed expandable as

$$
\begin{aligned}
H & =H_{0}+\sum_{n} \frac{\epsilon^{n}}{n !} H_{n} \\
H^{*}\left(-, \grave{u_{2}}, \grave{u_{3}} ; \grave{U} ; \epsilon\right) & =H_{0}^{*}\left(\grave{U_{1}}\right)+\sum_{n} \frac{\epsilon^{n}}{n !} H_{n}^{*}\left(-, \grave{u_{2}}, \grave{u_{3}} ; \grave{U}\right) \\
H^{* *}(-; \grave{U} ; \epsilon) & =H_{0}^{* *}(\grave{\dot{U}})+\sum_{n} \frac{\epsilon^{n}}{n !} H_{n}^{* *}(-; \grave{U}) \\
W(\grave{u} ; \grave{U} ; \epsilon) & =\sum_{n} \frac{\epsilon^{n}}{n !} W_{n+1}(\grave{u} ; \grave{U}) \\
W^{*}\left(-, \grave{\grave{u_{2}}}, \grave{\grave{u_{3}}} ; \grave{\dot{U}} ; \epsilon\right) & =\sum_{n} \frac{\epsilon^{n}}{n !} W_{n+1}\left(-, \grave{u_{2}}, \grave{\left.\grave{u_{3}} ; \grave{U}\right)}\right.
\end{aligned}
$$

\section{Elimination of Short period terms}

Using the transformation equations we have the following basic identities

$$
\begin{aligned}
H_{0}^{*} & =H_{0} \\
H_{n}^{*} & =\widetilde{H}_{n}+\left(H_{0} ; W_{n}\right) \\
\widetilde{H}_{n} & =H_{n}+\sum_{j=1}^{n-1}\left\{\left(\begin{array}{c}
n-1 \\
j-1
\end{array}\right)\left(H_{n-j} ; W_{j}\right)+\left(\begin{array}{c}
n-1 \\
j
\end{array}\right) G_{j} H_{n-j}^{*}\right\}
\end{aligned}
$$

Let $u_{1}$ be the fast variable in $H$. We choose $H_{n}^{*}$ to be the average of $\widetilde{H}_{n}$ over $u_{1}$; i. e.

$$
H_{n}^{*}=<\widetilde{H}_{n}>_{u_{1}}
$$

So that the periodic term is 


$$
P_{n}=\widetilde{H}_{n}-H_{n}^{*}=\left(H_{0} ; W_{n}\right)
$$

from which

$$
W_{n}=\left(\frac{\partial H_{0}}{\partial U_{1}^{1}}\right)^{-1} \int P_{n} d \grave{u}_{1}
$$

After determining the generator, the elements of the transformation and its inverse are determined by

$$
\begin{aligned}
u=\grave{u}+\sum_{n=1}^{2} \frac{J_{2}^{n}}{n !} \grave{u}^{(n)} \quad, \quad U=\grave{U}+\sum_{n=1}^{2} \frac{J_{2}^{n}}{n !} \grave{U}^{(n)} \\
\grave{u}=u+\sum_{n=1}^{2} \frac{J_{2}^{n}}{n !} u^{(n)}(u, U) \quad, \quad \grave{U}=U+\sum_{n=1}^{2} \frac{J_{2}^{n}}{n !} U^{(n)}(u, U)
\end{aligned}
$$

where

and

$$
\begin{aligned}
& \grave{u}^{(1)}=\frac{\partial W_{1}}{\partial \grave{U}} \quad, \quad \grave{u}^{(2)}=\frac{\partial W_{2}}{\partial \grave{U}}+L_{1} \grave{u}^{(1)} \\
& \grave{U}^{(1)}=-\frac{\partial W_{1}}{\partial \grave{u}} \quad, \quad \grave{U}^{(2)}=\frac{\partial W_{2}}{\partial \grave{u}}+L_{1} \grave{U}^{(1)}
\end{aligned}
$$

where $L_{1}$ is the Lie derivative.

$$
\begin{array}{ccc}
u^{(1)}=-\grave{u}^{(1)} & , \quad u^{(2)}=-\grave{u}^{(2)}+2 L_{1} \grave{u}^{(1)} \\
U^{(1)}=-\grave{U}^{(1)} & , \quad U^{(2)}=-\grave{U}^{(2)}+2 L_{1} \grave{U}^{(1)}
\end{array}
$$

Note that in the last two equations the right hand sides are evaluated at $\grave{u}=u$ and $\grave{U}=U$.

\section{A. Elimination of Long period terms}

The second transformation to eliminate the long period terms proceeds in exactly the same way but replacing $(u, U)$ by $(\grave{u}, \grave{U}) ;(\grave{u}, \grave{U})$ by $(\grave{\grave{u}}, \grave{U}) ; H$ by $H^{*} ; H^{*}$ by $H^{* *}$ and $W$ by $W^{*}$

\section{SOLUTION OF THE TRANSLATIONAL ROTATIONAL MOTION}

In this section the translational rotational motion is solved using the Lie technique. As discussed in the previous section, there will be two successive canonical transformations in order to eliminate the short and long period terms in succession. secular and periodic terms will be retained up to $O\left(J_{2}^{3}\right)$ and $O\left(J_{2}^{2}\right)$ respectively.

\section{A. Short Period transformation}

we proceed to eliminate the short period terms (those depending on $l, l_{a}$ and $g_{a}$ ) from the Hamiltonian in equation (26)

1) Zero Order: Using equation (32) and equation (27) we find that :

$$
\mathcal{H}_{0}^{*}=\mathcal{H}_{0}=-\frac{\mu^{2}}{2 L^{2}}+\frac{1}{2}\left(\frac{1}{I_{3}}-\frac{1}{I_{1}}\right) L_{a}^{2}+\frac{1}{2 I_{1}} G_{a}^{2}
$$

where all the variables in the right hand side are understood to be single primed, but the primes are removed from now on for simplicity.

2) First Order: From equation (32) we have :

$$
\mathcal{H}_{1}^{*}=\widetilde{\mathcal{H}}_{1}+\left(\mathcal{H}_{0} ; W_{1}\right)
$$

and

$$
\widetilde{\mathcal{H}}_{1}=\mathcal{H}_{1}=\frac{1}{4} \frac{A_{2}}{L^{6}}\left(\frac{a}{R_{c}}\right)^{3}\left[\left(3 s^{2}-2\right)-3 s^{2} \cos (2 f+2 g)\right]
$$

we then choose $\mathcal{H}_{1}^{*}$ to be the secular part of $\widetilde{\mathcal{H}}_{1}$. Thus we take the average of $\widetilde{\mathcal{H}}_{1}$ over the fast angles.

$$
\mathcal{H}_{1}^{*}=<\widetilde{\mathcal{H}}_{1}>_{l, l_{a}, g_{a}}=\frac{1}{8 \pi^{3}} \int_{0}^{2 \pi} \int_{0}^{2 \pi} \int_{0}^{2 \pi} \widetilde{\mathcal{H}}_{1} d l_{a} d g_{a} d l
$$

That is

$$
\mathcal{H}_{1}^{*}=\frac{1}{4} \frac{A_{2}}{L^{3} G^{3}}\left(1-3 \frac{H^{2}}{G^{2}}\right)
$$


The Periodic part is given as

$$
P=\widetilde{\mathcal{H}}_{1}-\mathcal{H}_{1}^{*}=\left(W_{1} ; \mathcal{H}_{0}\right)=\frac{1}{4} \frac{A_{2}}{L^{6}}\left(\frac{a}{R_{c}}\right)^{3}\left[\left(1-3 \frac{H^{3}}{G^{3}}\right)-3 s^{2} \cos (2 f+2 g)\right]-\frac{1}{4} \frac{A_{2}}{L^{3} G^{3}}\left(1-3 \frac{H^{2}}{G^{2}}\right)
$$

Since $P$ is independent of $l_{a}$ and $g_{a}$, we have only $\frac{\partial W_{1}}{\partial l}$ not vanishing.

thus

$$
\begin{aligned}
W_{1}=\frac{L^{3}}{\mu^{2}} \int P d l= & \frac{1}{4} \frac{A_{2}}{\mu^{2} G^{3}}\left(\left(1-3 \frac{H^{3}}{G^{3}}\right)(f-l+e \sin (f))\right. \\
& \left.-3 s^{2}\left[\frac{1}{2} \sin (2 f+2 g)+\frac{e}{2}\left(\frac{1}{3} \sin (3 f+2 g)+\sin (f+2 g)\right)\right]\right)
\end{aligned}
$$

3) Second order: Again we use the perturbation equations.

The equations for the second order are :

$$
\begin{aligned}
& \mathcal{H}_{2}^{*}=\widetilde{\mathcal{H}}_{2}+\left(\mathcal{H}_{0} ; W_{2}\right) \\
& \widetilde{\mathcal{H}}_{2}=\mathcal{H}_{2}+\left(\mathcal{H}_{1}+\mathcal{H}_{1}^{*} ; W_{1}\right)
\end{aligned}
$$

Where $\mathcal{H}_{2}$ is given in equation(29) and the bracket $\left(\mathcal{H}_{1}+\mathcal{H}_{1}^{*} ; W_{1}\right)$ is evaluated as:

$$
\begin{aligned}
\left(\mathcal{H}_{1}+\mathcal{H}_{1}^{*} ; W_{1}\right) & =-\frac{3 A_{2}^{2}}{16 \mu^{2} L^{4} G^{6}}\left(3 s^{2}-2\right)^{2}-\frac{3 A_{2}^{2}\left(4-5 s^{2}\right)}{16 \mu^{2} L^{3} G^{7}} \sum_{i=1}^{3} K_{i, 2}^{2} \cos (i f+2 g) \\
& +\frac{9 A_{2}^{2} s^{2}}{8 \mu^{2} L^{6} G^{4}}\left(4-5 s^{2}\right)(f-l)\left(\frac{a}{R_{c}}\right)^{3} \sin (2 f+2 g) \\
& +\frac{A_{2}^{2}}{256 e \mu^{2} L^{11} G^{8}}\left(\frac{a}{R_{c}}\right)^{2} \sum_{j=-1}^{2} \sum_{i=0}^{8} \widetilde{H}_{j, i} \cos (i f+2 j g)
\end{aligned}
$$

where $f$ is the true anomaly, $K_{i, 2}^{2}$ and $\widetilde{H}_{j, i}$ are functions of $e$ and $s$. Now $\widetilde{\mathcal{H}}_{2}$ is easily obtained. Now we take $\mathcal{H}_{2}^{*}$ in equation (46) to be the average of $\widetilde{\mathcal{H}}_{2}$

$$
\mathcal{H}_{2}^{*}=<\widetilde{\mathcal{H}}_{2}>_{l, l_{a}, g_{a}}=\frac{1}{8 \pi^{3}} \int_{0}^{2 \pi} \int_{0}^{2 \pi} \int_{0}^{2 \pi} \widetilde{\mathcal{H}}_{2} d l_{a} d g_{a} d l
$$

In performing this averaging we use Hansen coefficients to express functions of the true and eccentric anomaly in terms of the mean anomaly.

$$
\left(\frac{a}{R_{c}}\right)^{n} \cos (m f)=\sum_{k \geq 0} a_{k}^{-n, m}(e) \cos (k l)
$$

and

$$
\left(\frac{a}{R_{c}}\right)^{n} \sin (m f)=\sum_{k \geq 0} b_{k}^{-n, m}(e) \sin (k l)
$$

where the coefficients $a, b$ are Hansen coefficients that many procedures has been developed to evaluate[?]. Upon averaging of $\widetilde{\mathcal{H}}_{2}$ we have

$$
\begin{aligned}
& \mathcal{H}_{2}^{*}=\sum_{i=0}^{2} H_{i}^{*} \cos (i g)+\sum_{\substack{j=-2 \\
i=0,2,-2}}^{j=2} \widetilde{\widetilde{B}}_{0 i j 00}^{11} \cos \left(i g+j\left(h_{a}-h\right)\right)-\frac{\widetilde{Q} \mu R_{E}^{3}}{L^{2}} \times \\
& \sum_{\substack{j=-2 \\
k=-3}}^{\substack{j=4=3 \\
k=-2}}\left[F_{j k 0 n 00}^{11} \sin \left(j g+k h+n h_{a}\right)+\widetilde{F}_{j k 0 n 00}^{11} \cos \left(j g+k h+n h_{a}\right)\right]
\end{aligned}
$$


and the generator $W_{2}$ is given as

$$
\begin{aligned}
& W_{2}=\sum_{i=-\infty}^{\infty} \sum_{\substack{j=-2 \\
k=-3}}^{j=4, k=3} \sum_{m, r=-2}^{m, n=2}\left[F_{i j k m n r}^{22} \sin \left(i l+j g+k h+m g_{a}+n h_{a}+r l_{a}\right)\right. \\
& \left.+\widetilde{F}_{i j k m n r}^{22} \cos \left(i l+j g+k h+m g_{a}+n h_{a}+r l_{a}\right)\right]
\end{aligned}
$$

where $F_{j k 0 n 00}^{11}, \widetilde{F}_{j k 0 n 00}^{11}, F_{i j k m n r}^{22}$ and $\widetilde{F}_{i j k m n r}^{22}$ are functions of the action variables.

4) Third order:: The equations for the third order are

$$
\begin{aligned}
\mathcal{H}_{3}^{*}= & \widetilde{\mathcal{H}}_{3}+\left(\mathcal{H}_{0} ; W_{3}\right) \\
\widetilde{\mathcal{H}}_{3}= & \mathcal{H}_{3}+\sum_{j=1}^{2}\left\{\left(\begin{array}{c}
2 \\
j-1
\end{array}\right)\left(\mathcal{H}_{3-j} ; W_{j}\right)+\left(\begin{array}{c}
2 \\
j
\end{array}\right) G_{j} \mathcal{H}_{3-j}^{*}\right\} \\
= & \mathcal{H}_{3}+\left(\mathcal{H}_{2} ; W_{1}\right)+2 G_{1} \mathcal{H}_{2}^{*} \\
& +2\left(\mathcal{H}_{1} ; W_{2}\right)+G_{2} \mathcal{H}_{1}^{*} \\
= & \mathcal{H}_{3}+\left(\mathcal{H}_{2}+2 \mathcal{H}_{2}^{*}-\left(\mathcal{H}_{1}^{*} ; W_{1}\right) ; W_{1}\right)+\left(2 \mathcal{H}_{1}+\mathcal{H}_{1}^{*} ; W_{2}\right)
\end{aligned}
$$

The bracket $\left(\mathcal{H}_{2}+2 \mathcal{H}_{2}^{*}-\left(\mathcal{H}_{1}^{*} ; W_{1}\right) ; W_{1}\right)$ is evaluated as

$$
\begin{aligned}
\left(\mathcal{H}_{2}+2 \mathcal{H}_{2}^{*}-\left(\mathcal{H}_{1}^{*} ; W_{1}\right) ; W_{1}\right)= & \sum_{i=-\infty}^{\infty} \sum_{\substack{j=-4 \\
k=-3}}^{j=4, k=3} \sum_{m, n, r=-2}^{2} Q_{i j k m n r}^{11} \times \\
& \cos \left(i l+j g+k h+m g_{a}+n h_{a}+r l_{a}\right) \\
+ & \widetilde{Q}_{i j k m n r}^{11} \sin \left(i l+j g+k h+m g_{a}+n h_{a}+r l_{a}\right)
\end{aligned}
$$

and the bracket $\left(\mathcal{H}_{1}+2 \mathcal{H}_{1}^{*} ; W_{2}\right)$

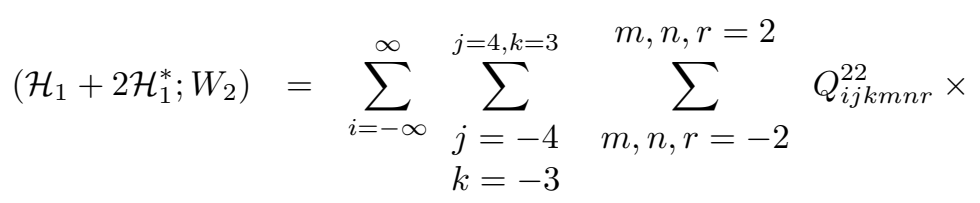

$$
\begin{aligned}
& \cos \left(i l+j g+k h+m g_{a}+n h_{a}+r l_{a}\right) \\
& \left.+\widetilde{Q}_{i j k m n r}^{22} \sin \left(i l+j g+k h+m g_{a}+n h_{a}+r l_{a}\right)\right]
\end{aligned}
$$

So that when using equation (30) we have

$$
\mathcal{H}_{3}^{*}=<\mathcal{H}_{3}+\left(\mathcal{H}_{2}+2 \mathcal{H}_{2}^{*}-\left(\mathcal{H}_{1}^{*} ; W_{1}\right) ; W_{1}\right)+\left(2 \mathcal{H}_{1}+\mathcal{H}_{1}^{*} ; W_{2}\right)>_{l, l_{a}, g_{a}}
$$

After performing the averaging we have

$$
\begin{aligned}
\mathcal{H}_{3}^{*}=-\frac{3 \mu^{6} R_{e}^{4}}{2 L^{10}}\left[\sum_{\substack{j=-2 \\
i=-2,0,2,4}}^{j=2} \widetilde{C}_{i j 00}^{11} a_{0}^{-5, i} \cos \left(i g+j\left(h_{a}-h\right)\right)\right] \\
+\sum_{\substack{j=-4 \\
k=-3}}^{2} \sum_{n=-2}^{2}\left(\widetilde{\widetilde{Q}}_{j k n}^{11} \cos \left(j g+k h+n h_{a}\right)+\widetilde{\widetilde{Q}}_{j k n}^{22} \sin \left(j g+k h+n h_{a}\right)\right) \\
\\
\left.+\sum^{j=3}\right)
\end{aligned}
$$

where $Q_{i j k m n r}^{11}, \widetilde{Q}_{i j k m n r}^{11}, Q_{i j k m n r}^{22}, \widetilde{Q}_{i j k m n r}^{22}, \widetilde{\widetilde{Q}}_{j k n}^{11}$ and $\widetilde{\widetilde{Q}}_{j k n}^{22}$ are all functions of the action variables and the previously computed coefficients. 


\section{B. Elements of Short Period transformation}

The elements of the transformation are obtained following the same procedures used by Kamel[?]. The equations are :

$$
\begin{gathered}
\grave{u}_{i}^{(1)}=\frac{\partial W_{1}}{\partial \grave{U}_{i}}=W_{1 i} \quad, \quad \grave{u}_{i}^{(2)}=\frac{\partial W_{2}}{\partial \grave{U}_{i}}+L_{1} \grave{u}_{i}^{(1)} \quad(i=1,2,3) \\
\grave{U}_{i}^{(1)}=-\frac{\partial W_{1}}{\partial \grave{u}_{i}}=W_{1,3+i} \quad, \quad \grave{U}_{i}^{(2)}=-\frac{\partial W_{2}}{\partial \grave{u}_{i}}+L_{1} \grave{U}_{i}^{(1)} \quad(i=1,2,3)
\end{gathered}
$$

where

$$
\begin{gathered}
L_{1} \grave{u}_{i}^{(1)}=\sum_{j=1}^{3}\left[\frac{\partial W_{1 i}}{\partial \grave{u}_{j}} W_{1 j}+\frac{\partial W_{1 i}}{\partial \grave{U}_{j}} W_{1,3+j}\right] \\
L_{1} \grave{U}_{i}^{(1)}=\sum_{j=1}^{3}\left[\frac{\partial W_{1, i+3}}{\partial \grave{u}_{j}} W_{1 j}+\frac{\partial W_{1, i+3}}{\partial \grave{U}_{j}} W_{1,3+j}\right]
\end{gathered}
$$

The derivatives of $W_{2}$ in the above expressions were already computed while evaluating Poisson brackets.

\section{Long Period Transformation}

In the new transformation we consider that the new Hamiltonian is expandable in powers of $J_{2}$ such that

$$
\mathcal{H}^{* *}=\mathcal{H}_{0}^{* *}\left(\grave{\grave{L}}, \grave{G}_{a}, \grave{L}_{a}\right)+\sum_{n=1}^{2} \frac{J_{2}^{n}}{n !} \mathcal{H}_{n}^{* *}\left(\grave{L}, \grave{G}, \grave{\dot{H}}, \grave{L}_{a}, \grave{G}_{a}, \grave{\grave{H}}_{a}\right)
$$

and the generator $W^{*}$ is expandable as

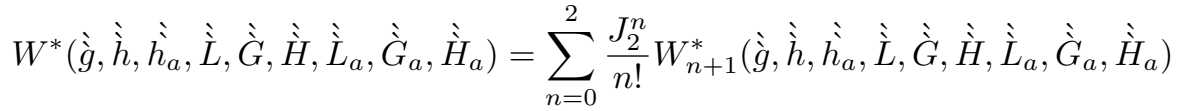

We use the same equations of transformation but now to transform the system associated with $\mathcal{H}^{*}$ to $\mathcal{H}^{* *}$

$$
\begin{aligned}
\mathcal{H}_{0}^{* *} & =\mathcal{H}_{0}^{*} \\
\mathcal{H}_{n}^{* *} & =\widetilde{\mathcal{H}}_{n}^{*}+\left(\mathcal{H}_{0}^{*} ; W_{n}^{*}\right) \\
\widetilde{\mathcal{H}}_{n}^{*} & =\mathcal{H}_{n}^{*}+\sum_{j=1}^{n-1}\left\{\left(\begin{array}{c}
n-1 \\
j-1
\end{array}\right)\left(\mathcal{H}_{n-j}^{*} ; W_{j}\right)+\left(\begin{array}{c}
n-1 \\
j
\end{array}\right) G_{j} \mathcal{H}_{n-j}^{* *}\right\}
\end{aligned}
$$

but since $W^{*}$ is a function of the slow variables only $\left(\grave{g}, \grave{\grave{h}}, \grave{h_{a}}\right)$ then the bracket $\left(\mathcal{H}_{0}^{*} ; W_{n}^{*}\right)$ vanishes, and the last two of equations (64) can be written in a single equation as

$$
\mathcal{H}_{n}^{* *}=\mathcal{H}_{n}^{*}+\sum_{j=1}^{n-1}\left\{\left(\begin{array}{c}
n-1 \\
j-1
\end{array}\right)\left(\mathcal{H}_{n-j}^{*} ; W_{j}\right)+\left(\begin{array}{c}
n-1 \\
j
\end{array}\right) G_{j} \mathcal{H}_{n-j}^{* *}\right\}
$$

Now we solve for different orders as before.

1) Zero Order:: The identity for the zero order is

$$
\mathcal{H}_{0}^{* *}=\mathcal{H}_{0}^{*}
$$

thus

$$
\mathcal{H}_{0}^{* *}=-\frac{\mu^{2}}{2 L^{2}}+\frac{1}{2}\left(\frac{1}{I_{3}}-\frac{1}{I_{1}}\right) L_{a}^{2}+\frac{1}{2 I_{1}} G_{a}^{2}
$$

where we will be omitting the double primes from now on for simplicity.

2) First order: From equation (65) the identity for the first order is

$$
\mathcal{H}_{1}^{* *}=\mathcal{H}_{1}^{*}
$$

from which

$$
\mathcal{H}_{1}^{* *}=\frac{1}{4} \frac{A_{2}}{L^{3} G^{3}}\left(1-3 \frac{H^{2}}{G^{2}}\right)
$$


3) Second order: : From (65) the identity for the first order is

$$
\begin{aligned}
\mathcal{H}_{2}^{* *} & =\mathcal{H}_{2}^{*}+\sum_{j=1}^{1}\left\{\left(\begin{array}{l}
1 \\
0
\end{array}\right)\left(\mathcal{H}_{1}^{*} ; W_{1}\right)+\left(\begin{array}{c}
1 \\
1
\end{array}\right) G_{1} \mathcal{H}_{1}^{* *}\right\} \\
& =\mathcal{H}_{2}^{*}+\left(\mathcal{H}_{1}^{*} ; W_{1}\right)+G_{1} \mathcal{H}_{1}^{* *} \\
& =\mathcal{H}_{2}^{*}+\left(\mathcal{H}_{1}^{*} ; W_{1}\right)+\left(\mathcal{H}_{1}^{*} ; W_{1}\right) \\
& =\mathcal{H}_{2}^{*}+2\left(\mathcal{H}_{1}^{*} ; W_{1}^{*}\right)
\end{aligned}
$$

so that

$$
\begin{gathered}
\mathcal{H}_{2}^{* *}=2\left(\mathcal{H}_{1}^{*} ; W_{1}^{*}\right)+\sum_{i=0}^{2} H_{i}^{*} \cos (i g)+\sum_{\substack{j=-2 \\
i=0,2,-2}}^{j=2} \widetilde{\widetilde{B}}_{0 i j 00}^{11} \cos \left(i g+j\left(h_{a}-h\right)\right)-\frac{\widetilde{Q} \mu R_{E}^{3}}{L^{2}} \times \\
\sum_{j=4, k=3}^{j=2} \sum_{j=-2}^{j=-2}\left[F_{j k 0 n 00}^{11} \sin \left(j g+k h+n h_{a}\right)+\widetilde{F}_{j k 0 n 00}^{11} \cos \left(j g+k h+n h_{a}\right)\right] \\
k=-3
\end{gathered}
$$

we perform the averaging over $\mathcal{H}_{2}^{*}$ and the secular part in the right hand side is then chosen to be $\mathcal{H}_{2}^{* *}$ that is

$$
\mathcal{H}_{2}^{* *}=H_{0}^{*}+\widetilde{\widetilde{B}}_{00000}^{11}-\frac{\widetilde{Q} \mu R_{E}^{3}}{L^{2}} \widetilde{F}_{000000}^{11}
$$

and then we evaluate $W_{1}$ from the remaining part as

$$
W_{1}=\sum_{\substack{j=-2 \\ k=-3}}^{\substack{j=4, k=3 \\ k=-2}}\left[R_{j k n}^{*} \cos \left(j g+k h+n h_{a}\right)+\widetilde{R}_{j k n}^{*} \sin \left(j g+k h+n h_{a}\right)\right]
$$

where as before the coefficients $H_{i}^{*}, \widetilde{\widetilde{B}}_{0 i j 00}^{11}, F_{j k 0 n 00}^{11}, \widetilde{F}_{j k 0 n 00}^{11}, R_{j k n}^{*}$ and $\widetilde{R}_{j k n}^{*}$ are functions of the action variables and the previously computed coefficients.

4) Third Order:: The equations of the third order are

$$
\begin{aligned}
\mathcal{H}_{3}^{* *} & =\widetilde{\mathcal{H}}_{3}^{*}+3\left(\mathcal{H}_{1}^{*} ; W_{2}^{*}\right) \\
\widetilde{\mathcal{H}}_{3}^{*} & =\mathcal{H}_{3}^{*}+\sum_{j=1}^{2}\left\{\left(\begin{array}{c}
2 \\
j-1
\end{array}\right)\left(\mathcal{H}_{3-j}^{*} ; W_{j}^{*}\right)+\left(\begin{array}{c}
2 \\
j
\end{array}\right) G_{j} \mathcal{H}_{3-j}^{* *}\right\} \\
& =\mathcal{H}_{3}^{*}+\frac{3}{2}\left(\mathcal{H}_{2}^{*}+\mathcal{H}_{2}^{* *} ; W_{1}^{*}\right)
\end{aligned}
$$

the bracket $\left(\mathcal{H}_{2}^{*}+\mathcal{H}_{2}^{* *} ; W_{1}^{*}\right)$ is evaluated as

$$
\left(\mathcal{H}_{2}^{*}+\mathcal{H}_{2}^{* *} ; W_{1}^{*}\right)=\sum_{\substack{j=-6 \\ k=-6}}^{\substack{j=8, k=6 \\ k=-4}}\left(S_{j k n}^{* 14} \cos \left(j g+k h+n h_{a}\right)+\widetilde{S}_{j k n}^{* 14} \sin \left(j g+k h+n h_{a}\right)\right)
$$

Proceeding, we then evaluate $\mathcal{H}_{3}^{* *}$ as

$$
\mathcal{H}_{3}^{* *}=<\widetilde{\mathcal{H}}_{3}^{*}>_{g, h, h_{a}}=-\frac{3 \mu^{6} R_{e}^{4}}{2 L^{10}}\left[\widetilde{C}_{0000}^{11} a_{0}^{-5,0}\right]+Q_{000000}^{11}+Q_{000000}^{22}+\frac{3}{2} S_{000}^{* 14}
$$


and

$$
\begin{aligned}
W_{2}^{*}= & -\frac{\mu^{6} R_{e}^{4}}{2 L^{10}} \sum_{\substack{k=-2 \\
j=-2,0,2,4}}^{k=2} \widetilde{C}_{j k}^{* 22} \sin \left(j g+k\left(h_{a}-h\right)\right) \\
+ & \frac{1}{3} \sum_{j=-4}^{j=4, k=3} \sum_{n=-2}^{2}\left[Q_{j k n}^{* 44} \sin \left(j g+k h+n h_{a}\right)-\widetilde{Q}_{j k n}^{* 44} \cos \left(j g+k h+n h_{a}\right)\right] \\
k=-3 & \\
+\frac{1}{2} \sum_{j=8, k=6}^{j=-6} \sum_{k=-4}^{j=-6} & \left.n S_{j k n}^{* 16} \sin \left(j g+k h+n h_{a}\right)-\widetilde{S}_{j k n}^{* 16} \cos \left(j g+k h+n h_{a}\right)\right]
\end{aligned}
$$

\section{Elements of Long Period transformation}

The elements of the transformation and its inverse maybe obtained from the equations of short period transformation, but with the replacement of $W_{n}$ by $W_{n}^{*},(u, U)$ by $(\grave{u}, \grave{U}), L_{n}$ by $L_{n}^{*}$ and $(\grave{u}, \grave{U})$ by $(\grave{u}, \grave{U})$.

\section{E. Secular perturbations and the computation of position and velocity}

The equations of motion are now reduced to

$$
\frac{d \grave{U}}{d t}=-\frac{\partial \mathcal{H}^{* *}}{\partial \grave{\grave{u}}}=0 \quad, \quad \frac{d \grave{\grave{u}}}{d t}=\frac{\partial \mathcal{H}^{* *}}{d \grave{\grave{U}}}=c
$$

where $c$ are arbitrary constants so that they admit the solution

$$
\grave{\grave{U}}=\grave{\grave{U}}_{0} \quad, \quad \grave{\grave{u}}=\grave{\grave{u}}_{0}+c t
$$

where the constants $\left(\grave{\dot{U}}_{0}, \grave{u}_{0}\right)$ are to be determined from the initial conditions.

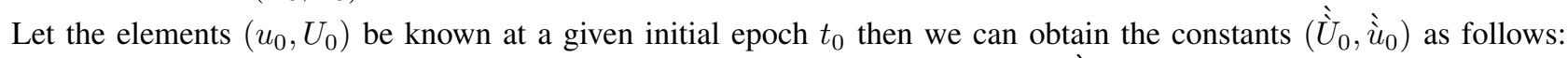

1 - From the elements of the transformation we can compute the initial values $\left(\grave{U}_{0}, \grave{u}_{0}\right)$ from

$$
\grave{u}_{0}=u_{0}+\sum_{n=1}^{2} \frac{J_{2}^{n}}{n !} u_{0}^{(n)} \quad, \quad \grave{U}_{0}=U_{0}+\sum_{n=1}^{2} \frac{J_{2}^{n}}{n !} U_{0}^{(n)}
$$

2- From the corresponding equations for the elements of the long period transformations

$$
\grave{\grave{u}}_{0}=\grave{u}_{0}+\sum_{n=1}^{2} \frac{J_{2}^{n}}{n !} \grave{u}_{0}^{(n)} \quad, \quad \grave{\grave{U}}_{0}=\grave{U}_{0}+\sum_{n=1}^{2} \frac{J_{2}^{n}}{n !} \grave{U}_{0}^{(n)}
$$

Now having determined $\grave{\dot{u}}_{0}$ and $\grave{U}_{0}$ we can evaluate $\mathcal{H}^{* *}=\mathcal{H}^{* *}(\grave{\dot{U}})$, and in turn the constants $c$ are now known.

To compute the position and velocity at any time $t$ we compute

$$
\grave{u}=\grave{\grave{u}}+\sum_{n=1}^{2} \frac{J_{2}^{n}}{n !} \grave{\grave{u}}^{(n)} \quad, \quad \grave{U}=\grave{\grave{U}}+\sum_{n=1}^{2} \frac{J_{2}^{n}}{n !} \grave{U}^{(n)}
$$

then

$$
u=\grave{u}+\sum_{n=1}^{2} \frac{J_{2}^{n}}{n !} \grave{u}^{(n)} \quad, \quad U=\grave{U}+\sum_{n=1}^{2} \frac{J_{2}^{n}}{n !} \grave{U}^{(n)}
$$

Having determined $(u, U)$ at time t, we compute the position, velocity, attitude and attitude motion of the spacecraft. 


\section{CONCLUSION}

In this paper we have obtained an analytical solution for the orbit-attitude motion of a charged spacecraft under the effect of Earth oblateness $\left(J_{2}\right)$ and Lorentz force through Hamiltonian framework. The problem is tackled by means of Lie perturbation technique. Two successive canonical transformations were performed in order to eliminate the short and long period terms in succession. secular and periodic terms were retained up to $O\left(J_{2}^{3}\right)$ and $O\left(J_{2}^{2}\right)$ respectively.

Acknowledgments

This project was supported financially by the Science and Technology Development Fund (STDF), Egypt, Grant No 1834.

\section{REFERENCES}

[1] Deprit, A. 1969: Celest. Mech. 1,12

[2] Dwidar,H.R. 2007,: "Analysis of the attitude motion of a satellite of an oblate earth". Ph.D. thesis, Cairo University

[3] Gurfil, P. et al. 2007: Regular and Chaotic Dynamics, 12,389

[4] Hughes, P.C. 1986:"Spacecraft attitude dynamics", John Wiley

[5] Kamel, A.A. 1969: Celest. Mech. 1,190

[6] Mohammed,Hani.M. 2012: "Attitude analysis of a rigid spacecraft". M.Sc. thesis, Cairo University

[7] Plummer, H.C. 1918 :" An introductory treatise on Dynamical Astronomy",Dover

[8] Tikhonov, A. A. 2002 : Cosmic Research. 40,171

[9] Tiknonov, A.A. and Petrov, K. G. 2002 : Cosmic Research. 40,219

[10] Tikhonov, A.A. 2003 : Cosmic Research. 41,69

[11] Zanardi, M.C. and De Moraes, R. V. 1999: Celest. Mech. 75,227 